\title{
iTimeline: Uma Abordagem Visual das Interações Sociais em Ambientes Virtuais de Aprendizagem Baseada no Modelo 3C de Colaboração
}

\author{
Francisco Soares de Sousa Neto - PPGI - IComp - UFAM \\ francisconeto@icomp.ufam.edu.br \\ Euler Vieira da Silva - IFAM Campus Maués \\ eulervieira@ifam.edu.br
}

\begin{abstract}
Resumo. As técnicas de Data Visualization (Dataviz) tem se destacado em diversos contextos de pesquisa, como por exemplo na Educação a Distância (EaD), no qual o Dataviz pode ser utilizado para auxiliar professores e alunos. Sendo assim, este artigo apresenta a ferramenta iTimeline (Linha do Tempo Interativa), uma ferramenta cujo objetivo é auxiliar professores que utilizam Ambientes Virtuais de Aprendizagem (AVA), fornecendo a eles um modelo visual que utiliza técnicas de Dataviz para traçar uma linha do tempo das interações dos alunos que ocorrem nos fóruns educacionais dos AVAs, auxiliando os professores na identificação e no acompanhamento das interações sociais. Neste artigo é apresentado a relação da iTimeline com o Modelo 3C de Colaboração.
\end{abstract}

Palavras-chave: Data Visualization; Linha do Tempo; AVA; Modelo 3C.

Abstract. The techniques of Data Visualization (Dataviz) has been highlighted in various contexts of research, as for example in Distance Education (EaD), in which the Dataviz can be used to assist teachers and students. Thus, this article presents the tool iTimeline (Time Line Interactive), a tool whose goal is to assist teachers of Virtual Learning Environments (AVA), providing them a visual model that uses techniques from Dataviz to provide a time line of interactions of students that occur in educational forums of AVAs, assisting teachers in the identification and monitoring of social interactions. This article is presented the relationship of iTimeline to Model $3 C$ for Collaboration.

Keywords: Data Visualization; Timeline; AVA; Model 3C.

\section{Introdução}

A Educação a Distância (EaD) vem ganhando espaço no campo educacional como uma modalidade de ensino-aprendizagem que supre as lacunas da educação tradicional, rompendo as barreiras do ensino presencial.

Em meio a esse contexto de EaD encontram-se os Ambientes Virtuais de Aprendizagem (AVA) que são plataformas educacionais constituídas por várias ferramentas de criação, gestão de atividades, comunicação, colaboração e interação (Cruz et al. 2012). Estes ambientes são desenvolvidos para o processo de ensino-aprendizagem, facilitando assim a comunicação entre alunos e professores (Neto et al. 2017). Porém, entre tantos benefícios que o AVA dispõe é importante sempre buscar novas formas de melhorar esse ambiente, a fim de tornar o AVA mais ágil e interessante aos seus usuários. Como por exemplo, o AVA não possui um suporte visual específico para o acompanhamento das interações dos alunos, o que impossibilita o professor de identificar rapidamente, 
coletar e analisar dados que são importantes para a promoção do ensino e da aprendizagem.

Segundo Neto et al. (2016), a análise das interações no processo do Ensino a Distância surge para ampliar a prática educacional pedagógica, e para que isso se torne uma realidade é necessário promover situações em que os alunos se defrontem com as diversas maneiras de aprender. Por isso, percebe-se que a interação social contribui no processo do conhecimento como construção do saber interativo, interpessoal e participativo

Pensando nisso, surgiu a ferramenta iTimeline (linha do tempo interativa) que utiliza de técnicas de Data Visualization (Dataviz) para elaborar e fornecer ao professor uma linha do tempo interativa com informações a respeito das interações dos alunos nas ferramentas mensagem e fórum de discussão do AVA, dessa forma, o professor terá de forma rápida o acesso aos dados interacionais.

Este artigo correlaciona os resultados obtidos da ferramenta iTimeline com o Modelo 3C de Colaboração, objetivando os aspectos coordenativos, comunicativos e cooperativos que acontecem entre os alunos e professores de um curso no AVA por intermédio da iTimeline.

Segundo Fuks et al. (2004) essas ferramentas interativas são utilizadas para facilitar no processo de ensino-aprendizagem e estimular a colaboração e a interação entre os participantes de um curso baseado na web e se fazem necessárias na Educação a Distância.

Para possibilitar um melhor detalhamento da proposta, este artigo foi dividido em 6 Seções. A Seção 1 descreve a introdução, em seguida a Seção 2 destaca a fundamentação teórica embasando a relevância desta pesquisa. A Seção 3 menciona os trabalhos relacionados a esta pesquisa. Na Seção 4 discorri sobre a ferramenta iTimeline apresentando a sua arquitetura. A Seção 5 e 6 apresentam os resultados obtidos e contribuições. Por fim a Seção 7, apresenta as considerações finais.

\section{Fundamentos Teóricos}

Nesta Seção será apresentada a fundamentação teórica na qual consiste na revisão dos trabalhos relacionados a esta pesquisa, auxiliando na compreensão do Estado da Arte em relação a esta temática.

\subsection{Data Visualization no Ensino a Distância}

Data Visualization é uma das áreas da tecnologia da informação que vem se destacando muito nos últimos anos e surge como uma metodologia moderna de como visualizar e analisar dados. Este método visual envolve a criação de um estudo representativo dos dados.

Masud et al. (2010), considera visualizações no domínio mais amplo de comunicação e define um modelo capaz de levar em conta o contexto em que visualizações atuam como ferramentas de comunicação, neste sentido, o autor identifica os dados e informações, como um contínuo conhecimento e sendo o elemento chave para uma estrutura, que considera a visualização como um processo e não como um produto.

De acordo com Tervakari et al. (2014), ferramentas analíticas visuais podem ajudar os professores a tomar decisões sobre estratégias pedagógicas, orientação educacional, ações e intervenções que podem ser usadas para promover e apoiar a participação e a atividade dos alunos. 
Entretanto, o objetivo principal de visualizar dados é comunicar e informar de forma clara e eficiente os usuários. A metodologia de visualização de dados ajuda os professores analisarem e raciocinarem acerca das informações possibilitando interpretações e tomadas de decisões rápidas.

\subsection{Modelo 3C de Colaboração}

Ellis et al. (1991) inicia o estudo sobre o Modelo 3C, identificando sistemas que dão suporte ao trabalho em grupo classificando-os em três dimensões (Fuks et al. 2011). Esta classificação deu suporte para a estruturação do Modelo 3C de Colaboração (comunicação, coordenação e cooperação) desenvolvido por Fuks et al. (2003). Diante deste modelo, destaca-se o trabalho em grupo, pois ajudam as pessoas a produzirem potencialmente melhores resultados do que trabalhando individualmente.

Para colaborar, os indivíduos trocam informações (se comunicam), organizamse (se coordenam) e operam em conjunto num espaço compartilhado (cooperam). As trocas ocorridas durante a comunicação geram compromissos, pois são gerenciados pela coordenação, que por sua vez organiza e dispõe as tarefas que são executadas na cooperação. Ao cooperar os indivíduos têm necessidade de se comunicar para renegociar e para tomar decisões sobre situações não previstas inicialmente. Isto mostra o aspecto cíclico da colaboração (Fuks et al. 2003).

Analisando as aplicações colaborativas, um dos primeiros sistemas de classificação a ficar amplamente conhecido foi à taxonomia espaço-tempo de (DeSanctis et al. 1987). Outra proposta foi a classificação dos sistemas colaborativos ao nível da aplicação, sendo enunciadas 7 classes de aplicações colaborativas (Ellis et al. 1991). Estas classes foram analisadas em função do grau de suporte à comunicação, coordenação e cooperação, sendo posicionadas no espaço triangular apresentado na Figura 1.

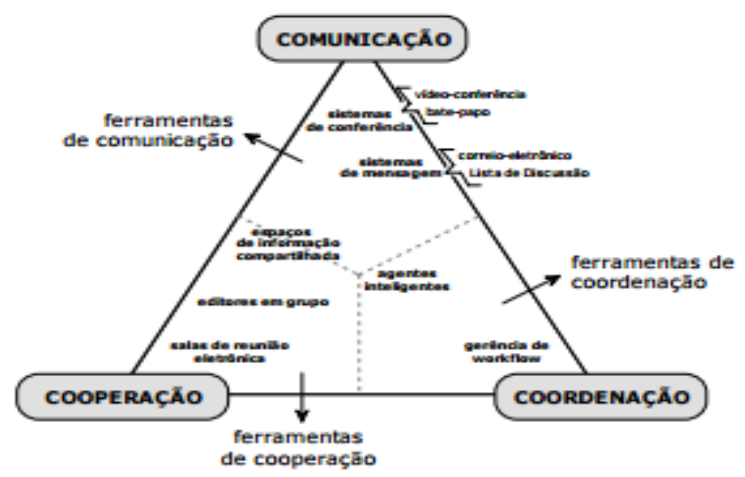

Figura 1: Classificação 3C dos sistemas colaborativos Pimentel et al. (2006).

- Comunicação é a ação de trocar mensagens para que haja entendimento comum das ideias discutidas. Na Colaboração, a Comunicação é direcionada para a ação. Os participantes de um grupo de trabalho trocam ideias e pontos de vista, apresentam seus argumentos e discutem com a finalidade de realizar negociações, tomar decisões e firmar compromissos. Coordenação é a ação de dispor segundo uma certa ordem e método, organizar, arranjar (Fuks et al. 2003). 
- Coordenação também está associada à ideia ao planejamento. Fazem parte da Coordenação a identificação de objetivos, o mapeamento dos objetivos em tarefas, a seleção dos participantes e a distribuição das tarefas. Através da Coordenação, as tarefas atribuídas aos diferentes participantes são realizadas na ordem e no tempo previstos e de acordo com os objetivos e restrições determinados (Fuks et al. 2002).

- Cooperação é a ação de operar simultaneamente. Ao cooperarem, os participantes do grupo produzem, modificam e utilizam de maneira compartilhada um conjunto informações e artefatos reais ou virtuais, tais como um documento, uma planilha, ou a peça de um carro. A Cooperação é então esta operação conjunta dos membros do grupo no espaço compartilhado a fim de que as atividades possam ser realizadas (Fuks et al. 2002).

\section{Trabalhos Relacionados}

Atualmente trabalhos analisam a interação dos usuários dentro do AVA, e outros além de realizar tal análise, também incorporam algumas técnicas de visualização de dados a esses ambientes a fim de torná-los mais interativos. Os trabalhos a seguir correlacionam este trabalho.

No trabalho de Bakharia e Dawson (2011), é apresentada a ferramenta SNAPP, tendo como finalidade, possibilitar aos instrutores do AVA a visualização e evolução das relações dos participantes dentro do fórum de discussão em forma de sociograma e disponibiliza em tempo real as métricas de rede social que foram aplicadas na disciplina. Permitindo que os padrões de interação sejam analisados e caso necessário, interferidos pelo instrutor do curso.

No trabalho Bassani (2006), é apresentado uma reflexão sobre a questão da interação em Ambientes Virtuais de Aprendizagem, focalizando-se na modelagem e implementação de uma ferramenta computacional capaz de mapear as interações potencializadas nestes espaços. Logo, busca, por um lado, investigar se o mapeamento das interações que se constituem entre os sujeitos participantes de um curso em um Ambiente Virtual de Aprendizagem pode auxiliar o professor no processo de avaliação das atividades desenvolvidas no ambiente.

Já o trabalho de Pimentel et al. (2006), mostra a importância do Modelo 3C em diferentes etapas do processo de desenvolvimento de sistemas colaborativos, destacando a importância do Modelo 3C nas análises do groupware a ser desenvolvido; no estabelecimento do foco para o desenvolvimento de sucessivas versões do groupware num processo iterativo e investigativo; e para o desenvolvimento de arquitetura e componentes $3 \mathrm{C}$.

No trabalho de Catrambone et al. (2009), é apresentada uma ferramenta de visualização dinâmica - denominada de Command Post of the Future (CPOF) - que suporta a tomada de decisão colaborativa em unidades táticas. O sistema utiliza um espaço de trabalho personalizável com base nas necessidades do usuário, em vez de um formato estático.

Por fim, o trabalho de Fuks et al. (2005), analisa as interações que ocorrem nos fóruns de discussões, possibilitando uma melhor coordenação dos professores para com os alunos. Neste trabalho também é investigado a possibilidade de como melhorar o 
apoio à coordenação de um fórum usando dispositivos móveis para mitigar as dificuldades do professor em seguir o status de um fórum.

Esta proposta se diferencia das demais pesquisas mencionadas anteriormente, pelo fato de permitir aos professores selecionar informações interacionais dos alunos e visualizá-las em forma de linha de tempo e de acordo com as opções disponibilizadas pela ferramenta. Propomos um processo de acompanhamento interativo, dinâmico e cronológico das interações, possibilitando ao professor utilizar táticas pedagógicas quando achar necessário diante dos dados que lhe são apresentados. Além disso, esta abordagem prevê o uso de mecanismos de colaboração, pois existem momentos que os professores e alunos formam um clico colaborativo no ambiente.

\section{A Proposta}

A arquitetura do sistema é composta como ilustra a Figura 2. O processo de desenvolvimento apresentado é baseado no modelo arquitetural que esboça uma visão geral da ferramenta. Esta abordagem tem a vantagem de combinar técnicas de Dataviz e de Sistemas Multiagente, integrados em Ambientes Virtuais de Aprendizagem (AVA), proporcionando a interatividade e a dinamicidade que a ferramenta dispõe para o acompanhamento das interações dos alunos.
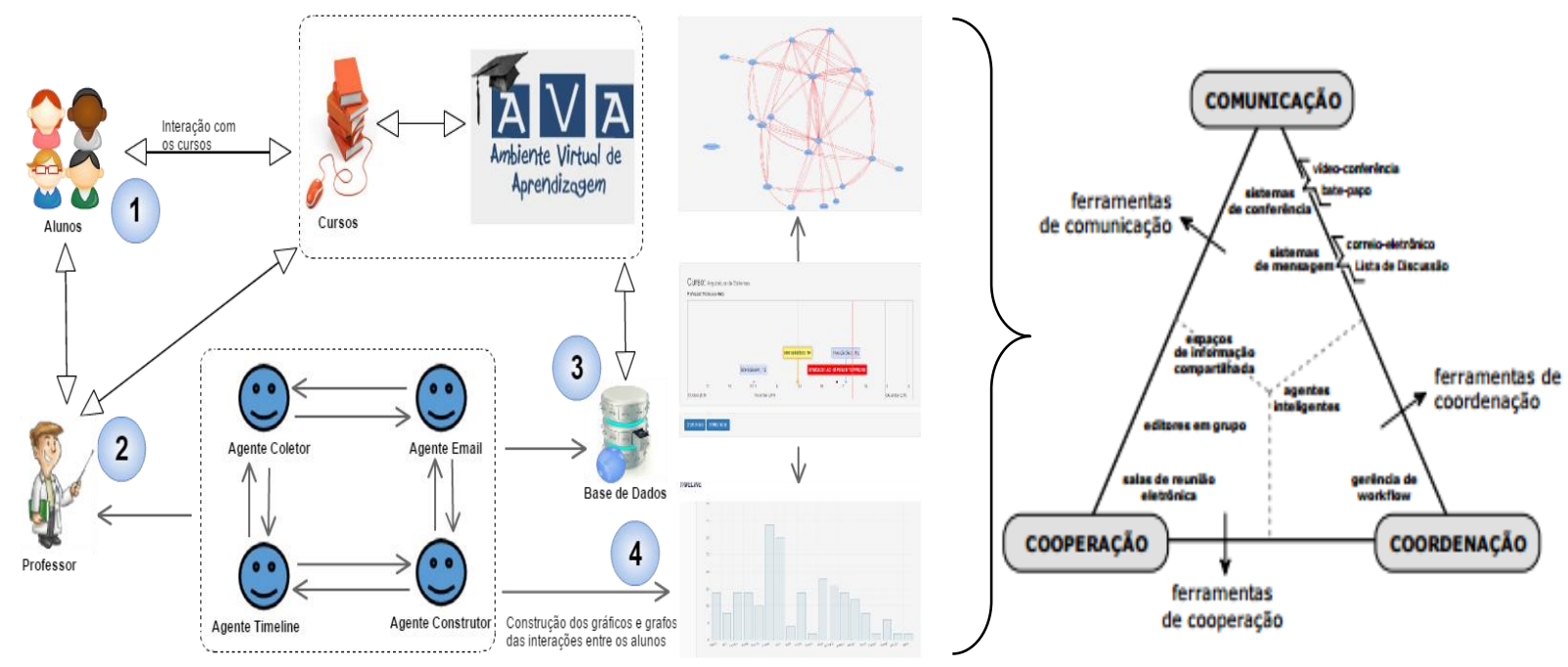

Figura 2: Visão arquitetural da ferramenta.

A iTimeline disponibiliza alguns recursos visuais para que os dados possam ser visualizados, os quais podem ser utilizados em um curso no AVA para identificação e análise das interações dos alunos. Esses recursos são disponibilizados ao professor no decorrer de um curso a distância.

De acordo com a visão arquitetural da ferramenta apresentada na Figura 2, pode-se observar o seguinte:

1. Em um AVA pode-se ter inúmeros cursos de diversas áreas do conhecimento, os mesmos têm como propósito transmitir o conhecimento. Os alunos e os professores participam dos cursos no ambiente virtual, o professor nesta etapa desempenha um papel muito importante, o mesmo realiza a matrícula dos alunos, cadastra os cursos, posta as avaliações, monitora o ambiente entre outras ações.

2. O professor pode acompanhar em tempo real o que está acontecendo no ambiente, assim ele poderá obter informações sobre o andamento da turma 
e, consequentemente, poderá interagir com seus alunos no momento em que achar oportuno. As interações analisadas pelos agentes integrados na ferramenta iTimeline, acontecem nas mensagens e nos fóruns de discussões no AVA.

3. As informações armazenadas no banco de dados são identificadas e coletadas pelo Agente Coletor, o mesmo é o responsável por informar o Agente Timeline (construtor da linha do tempo) a estruturar os dados cronologicamente. Cada um dos agentes tem o seu papel. A comunicação entre os agentes funciona de acordo com os dados que estão sendo requisitado pelo professor.

4. Os dados identificados e estruturados em modelos visuais baseados em grafos e gráficos pelo Agente Construtor na linha tempo. São apresentados os diversos estágios das interações entre os alunos, assim, simplificando o trabalho do professor no acompanhamento das interações no ambiente. A ferramenta possui um mecanismo de feedback composto por um agente denominado Agente Email, o qual identifica os alunos com pouca ou nenhuma interação, e envia um e-mail para o professor apresentando os dados interacionais.

\section{Contribuição da iTimeline}

Atualmente, trocar informações, se comunicar e interagir, tornam indispensáveis no processo de ensinar e aprender por meio de ambientes virtuais de aprendizagem. Segundo Fuks et al. (2003), para colaborar, os indivíduos têm que trocar informações (se comunicar), organizar-se (se coordenar) e operar em conjunto num espaço compartilhado (cooperar). As trocas de informações ocorridas durante a comunicação geram compromissos que são gerenciados pela coordenação, na qual organiza e dispõe as tarefas que são executadas na cooperação. Ao cooperar, os indivíduos têm necessidade de se comunicar para renegociar e para tomar decisões, assim formando o processo colaborativo.

Se aprender a colaborar torna-se um dos principais objetivos no processo educacional, onde os estudantes tornaram-se proficientes em colaboração (Burton et al. 1997). Um sistema colaborativo geralmente integra um conjunto de ferramentas para colaboração dos participantes (Fuks et al. 2003).

Diante dos conceitos abordados, a ferramenta iTimeline colabora significativamente no processo de ensinar e aprender, pois a mesma pode auxiliar o professor na identificação das interações sociais dos alunos em um curso a distância. A ferramenta possui uma infraestrutura dinâmica e interativa, que auxilia o professor na promoção do ensino e da aprendizagem. Além disso, oferece algumas funcionalidades aos professores, como por exemplo: em uma linha do tempo com as interações em tempo real, coletadas nos chats e fóruns de discussão no AVA, podendo exibi-las por meio dos recursos visuais anteriormente abordados. Para entendermos melhor a linha do tempo interativa, a mesma está destacada na Figura 3. 


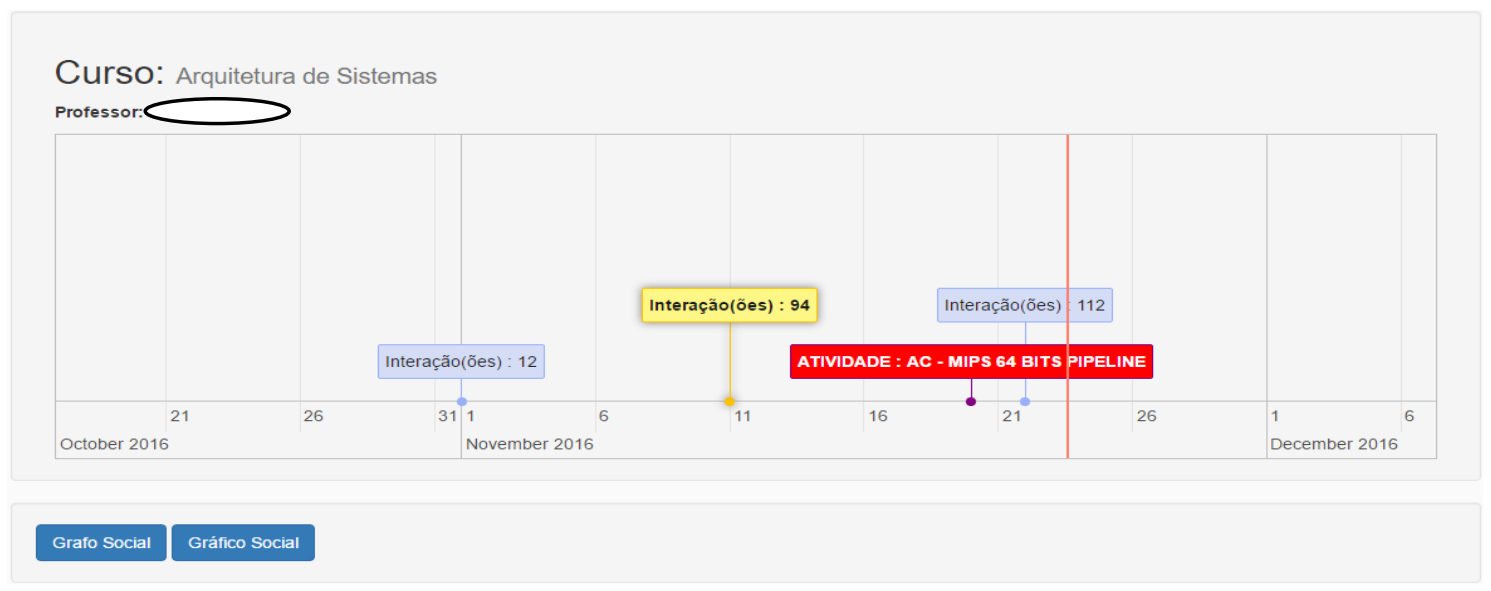

Figura 3: Linha do tempo identificando as interações.

Neste contexto, surge o questionamento: como a ferramenta colabora no processo de ensino-aprendizagem? Colabora por fornecer ao professor um monitoramento das interações ocorrentes no ambiente. Assim por meio desse acompanhamento, o professor poderá diante das informações, intervir ou não pedagogicamente. A intervenção pode acontecer quando o professor de posse dos dados, perceber que a interação não está acontecendo. Na Figura 4 são demonstrados os recursos visuais que a ferramenta disponibiliza. Neste propósito, serão explicados cada um dos recursos visuais.

Grafos: esta modalidade de exibição dos dados proporcionado pela ferramenta, ajuda o professor na verificação e na identificação de grupos. Explicando melhor este modelo de visual das interações dos alunos, o grafo possui o nó (identifica o aluno) e aresta (representa as ligações entre os alunos).

Gráficos: já com esta opção de visualizar as interações, o professor pode analisar os índices quantitativos individualmente de cada aluno. Com a possibilidade de analisar os dados, o professor poderá identificar o aluno com pouca interação no ambiente.

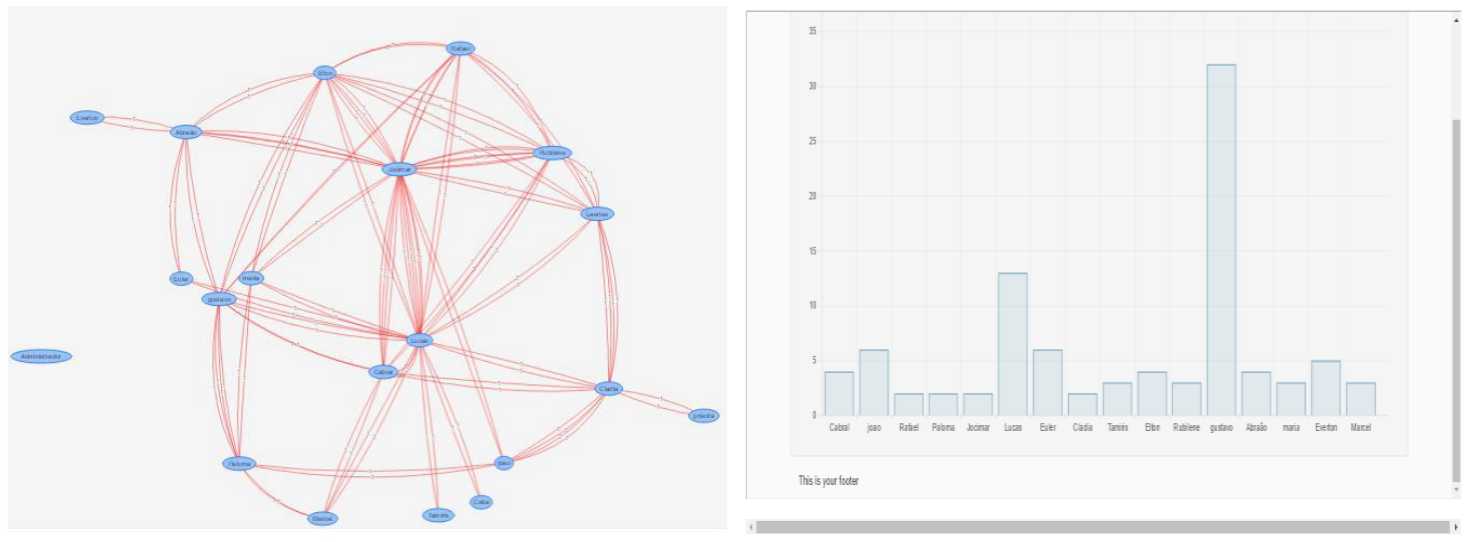

Figura 4: Visualização das interações (lado esquerdo grafo) e (lado direito gráfico).

Além da iTimeline propiciar a exibição dos dados por meio de grafos e gráficos, a mesma fornece por intermédio de e-mail um feedback do quadro interacional dos alunos. Porém, é importante salientar que o e-mail é enviado automaticamente pela ferramenta, onde professor pode identificar os alunos com pouca interatividade. Esse mecanismo de feedback foi introduzido na ferramenta para facilitar no monitoramento da turma. 


\subsection{Contribuição Comunicativa}

A iTimeline projeta-se como uma ferramenta comunicativa mediada por meio de técnicas de Data Visualization e Sistemas Multiagente visando suporte às interações, subsidiando o professor no acompanhamento cronológico dos diversos momentos em que as mesmas acontecem no ambiente. Para acontecer a disseminação do conhecimento de forma coletiva e colaborativa, é imprescindível que os alunos se comuniquem e interrelacione entre si. Porém, essa troca de informações envolve os alunos na promoção do ensino e da aprendizagem.

Portanto o relacionamento interativo, advindo dessa comunicação, torna-se uma condição essencial para a tomada de decisão dos professores, pois diante dos diferentes estágios interacionais ocorridos no AVA. A comunicação no ambiente educacional viabiliza o processo cooperativo, no entanto, esse processo comunicativo torna-se uma condição necessária entre os alunos nos ambientes educacionais virtuais.

\subsection{Contribuição Coordenativa}

Com posse da iTimeline, o professor consegue realizar o acompanhamento das interações, coordenando a turma e promovendo a melhoria na inter-relação entre os membros. Nesse sentido, a coordenação pode acontecer por parte do professor, coordenando o processo de ensino e aprendizagem entre os alunos.

O professor pode elaborar um planejamento de ensino diante da visualização das informações, além de associar os recursos disponibilizado pela ferramenta iTimeline para coordenar a interação na promoção do conhecimento dos alunos. Também tornamse função do professor intervir e incentivar a participação dos alunos. Canepa et al. (2009), afirma que a coordenação permite o planejamento e a gerência das atividades durante o trabalho, maximizando as atividades do grupo.

\subsection{Contribuição Cooperativa}

O AVA oferece um conjunto de ferramentas para o gerenciamento de seus membros, bem como ferramentas de suporte a comunicação entre os mesmos, disponibilizando mecanismos para o trabalho cooperativo e colaborativo (Pinto et al. 2002). A cooperação é a ação de trabalhar simultaneamente num espaço compartilhado. Quando cooperam, os participantes buscam realizar uma tarefa, produzindo, modificando artefatos ou organizando informações (Fuks et al. 2005).

Os ambientes virtuais de aprendizagem são constituídos por ferramentas computacionais que intermediam práticas colaborativas e cooperativas entre os participantes. Nesse sentido, a ferramenta iTimeline pode auxiliar no processo de cooperação, diminuindo as barreiras do espaço-tempo, ampliando a cooperação e a sinergia entre os alunos. $\mathrm{O}$ ambiente de trabalho virtual se configura como um espaço de comunicação e mediatização propício para desencadear a cooperação entre professoraluno numa dinâmica de interação entre as pessoas e os conteúdos culturalmente selecionados (Valente et al. 2003).

Os registros de mensagens nas ferramentas chats e fóruns de discussão no AVA, são identificadas e estruturadas pela ferramenta iTimeline. Dessa maneira tornase possível perceber o processo cooperativo no ambiente, pois são onde os alunos discutem sobre as atividades, ajudando uns aos outros. Portanto, dessa maneira, a memória do grupo é preservada nas ideias, fatos, questões, pontos de vista, conversações, discussões, decisões, etc., que são recuperáveis, possibilitando 
reconstituir o histórico da colaboração e o contexto onde a aprendizagem aconteceu (Kanselaar et al. 2003).

\section{Considerações Finais}

A interação entre alunos tem um papel muito importante na aprendizagem, possibilitando a troca de conhecimento e ideias. A iTimeline dá suporte no monitoramento das interações contribuindo para promoção da aprendizagem colaborativa. Entretanto, os ambientes virtuais de aprendizagem geralmente não oferecem esse suporte computacional cronológico específico para apoiar o professor acompanhamento nas relações dos alunos.

Sob a perspectiva de uma ferramenta visual para auxiliar no processo de ensino-aprendizagem. Quando utilizada no contexto educacional na modalidade a distância, a iTimeline torna-se uma ferramenta promissora no acompanhamento das informações visuais e pode ser utilizada para promover a interação do aluno colaborando no ensino e na aprendizagem. Os resultados apresentados neste artigo ajudam a mediar o processo de identificação das diversas situações interacionais. Apesar deste artigo ter foco nas interações dos alunos em AVA. Vale ressaltar também que a ferramenta torna-se apropriada para identificar e apresentar as informações de maneira visual. Portanto, com base nessa pesquisa foi proposto uma nova abordagem que visa permitir ao professor selecionar e visualizar informações sobre as interações no contexto da Educação a Distância.

\section{Referências}

Bakharia, Aneesha; Dawson, Shane. SNAPP: a bird's-eye view of temporal participant interaction. In: Proceedings of the 1st international conference on learning analytics and knowledge. ACM, 2011. p. 168-173.

Bassani, Patrícia Brandalise Scherer. Mapeamento das interações em ambiente virtual de aprendizagem: uma possibilidade para avaliação em educação a distância. 2006.

Bruno S. Nascimento, Marcos R.S. Borges, Adriana S. Vivacqua. - Uma Abordagem para Seleção e Visualização Colaborativa de Informações em Situações de Emergência. Simpósio Brasileiro de Sistemas Colaborativos SBSC, Curitiba, PR, Brasil, 2014.

Burton, Mark; Brua, Paul; Treasure-Jones, Tamsin. Splitting the Collahorative Atom: How to Support Learning ahout Collahorstion. Artificial intelligence in education: Knowledge and media in learning systems, v. 39, p. 135, 1997.

Canepa, K., Fuks, H., Carvalho, G. Training in Requirements by Collaboration: Branching Stories in Second Life. In: Simpósio Brasileiro de Sistemas Colaborativos (SBSC), Fortaleza - CE, 2009.

Catrambone, Richard; Wampler, Richard L.; Bink, Martin L. Determining a critical-skill hierarchy for Command Post of the Future (CPOF). Georgia Inst Of Tech Atlanta, 2009.

Cruz, R. M. et al. Abordagem conceitual de um sistema multiagente de recomendação de Objetos de Aprendizagem aos alunos no ambiente moodle. In: XVIII Congresso Internacional de Educação a Distância, Anais da ABED. 2012.

Neto, Francisco S. S, José Francisco Netto, and Dhanielly Lima. "iTimeline: Uma Ferramenta Visual para Identificação e Acompanhamento das Interações Sociais em Ambientes Virtuais de Aprendizagem." Brazilian Symposium on Computers in 
Education (Simpósio Brasileiro de Informática na Educação-SBIE). Vol. 28. No. 1. 2017.

Desanctis, Gerardine; Gallupe, R. Brent. A foundation for the study of group decision support systems. Management science, v. 33, n. 5, p. 589-609, 1987.

Ellis, Clarence A.; Gibbs, Simon J.; Rein, Gail. Groupware: some issues and experiences. Communications of the ACM, v. 34, n. 1, p. 39-58, 1991.

Fuks, H., Gerosa, M. A., Raposo, A. B., \& Lucena, C. D. O modelo de colaboração 3C no ambiente AulaNet. Informática na Educação: Teoria e Prática, 7(1), 25-48 2004.

Fuks, H., Gerosa, M.A. \& Lucena, C.J.P. "The Development and Application of Distance Learning on the Internet", Open Learning Journal, V.17, N.1, pp. 23-38, 2002.

Fuks, H., Raposo, A. B. And Gerosa, M. A. Do Modelo de Colaboração 3C à Engenharia de Groupware, In Anais do Simpósio Brasileiro de Sistemas Multimídia e Web - Webmidi, 2003.

Fuks, H., Raposo, A. B., Gerosa, M. A., Pimentel, M., Filippo, D. and Lucena, C. J. P. de. Teorias e modelos de colaboração. In Fuks, H.; Pimentel, M. Sistemas Colaborativos. 1. ed. Elsevier, 416p, 16-33, 2011.

Fuks, H., Raposo, A. e Gerosa, M.A. "Engenharia de Groupware: Desenvolvimento de Aplicações Colaborativas", XXI Jornada de Atualização em Informática, Anais do XXII Congresso da Sociedade Brasileira de Computação, v.2, Cap. 3, ISBN 85-88442-24-8, pp 89-128, 2002.

Fuks, H., Raposo, A.B., Gerosa, M.A. \& Lucena, C.J.P. Applying the 3C Model to Groupware Development. International Journal of Cooperative Information Systems (IJCIS), v.14, n.2-3, Jun-Sep, World Scientific, ISSN 0218-8430, p.299-328, 2005.

Gerosa, M. A. Filippo, D. Pimentel, M. Fuks, H. Lucena, C.J.P. Is the unfolding of the group discussion off-pattern? Improving coordination support in educational forums using mobile devices. Computers and Education, v. 54, p. 528-544, 2010.

Kanselaar, G., Erkens, G., Andriessen, J., Prangsma, M., Veerman, A. \& Jaspers, J. Designing Argumentation Tools for Collaborative Learning, Visualizing Argumentation: Software Tools for Collaborative and Educational Sense-Making, Kirschner, P., Shum, S. and Carr, C. (Eds.), Cap 3, Springer-Verlag, London, 2003.

Masud, L. Valsecchi, C. Paolo, R. Donato, Caviglia, G. From Data to Knowledge: Visualizations as Transformation Processes Within the Data-Information-Knowledge Continuum. Proceedings of the International Conference on Information Visualisation, 2010 .

Pimentel, M., Gerosa, M. A., Filippo, D., Raposo, A., Fuks, H., \& Lucena, C. J. P. Modelo 3C de Colaboração para o desenvolvimento de Sistemas Colaborativos. Anais do III Simpósio Brasileiro de Sistemas Colaborativos, 58-67, 2006.

Pinto, S. C. C., Schlemmer, E., dos Santos, C. T., Pérez, C. C., \& Rheinheimer, L. R. Ava: Um ambiente virtual baseado em comunidades. In Brazilian Symposium on Computers in Education (Simpósio Brasileiro de Informática na Educação-SBIE) (Vol. 1, No. 1, pp. 31-38), 2002. 
Tervakari, S. Kirsi, K. Juho P. Jukka, O. Usefulness of Information Visualizations Based on Educational Data. IEEE Global Engineering Education Conference, EDUCON, 2014.

Valente, José Armando; Prado, Maria Elisabette B. Brito; Almeida, Maria Elizabeth Bianconcini de. Educação a distância via Internet. São Paulo: Avercamp. p. 149, 2003. 